EPJ Web of Conferences 112, 03006 (2016)

DOI: $10.1051 /$ epjconf/201611203006

(C) Owned by the authors, published by EDP Sciences, 2016

\title{
Frontiers of QCD with Precision nPDFs *
}

\author{
Aleksander Kusina $^{1, a}$, Florian Lyonnet ${ }^{2, b}$, Fredrick I. Olness ${ }^{2, c, d}$, and Ingo Schienbein ${ }^{1, e}$ \\ ${ }^{1}$ LPSC, Université Joseph Fourier/CNRS-IN2P3/INPG, UMR5821, Grenoble, F-38026, France \\ ${ }^{2}$ Southern Methodist University, Dallas, TX 75275, USA
}

\begin{abstract}
Searches for new physics will increasingly depend on identifying deviations from precision Standard Model (SM) predictions. Quantum Chromodynamics (QCD) will necessarily play a central role in this endeavor as it provides the framework for the parton model. However, as we move to higher orders and into extreme kinematic regions, we begin to see the full complexities of the QCD theory. Recent theoretical developments improve our ability to analyze both proton and nuclear PDFs across the full kinematic range. These developments are incorporated into the new nCTEQ15 PDFs, and we review these developments with respect to future measurements, and identify areas where additional effort is required.
\end{abstract}

\section{The Character and Consequences of $Q C^{1}$}

"QCD is our most perfect physical theory." 2 Its scope is wide ranging spanning nuclear physics, particle physics, cosmology, and astrophysics. It also contains a wealth of phenomena including: "radiative corrections, running couplings, confinement, spontaneous (chiral) symmetry breaking, anomalies, and instantons"[1].

QCD is both a force whose properties we want to study, and also a tool we can use to link experimentally measured hadronic cross sections to theoretically calculable cross sections in the context of the parton model. The basic statement of the parton model is: $\sigma_{A \rightarrow X}=f_{a / A} \otimes \widehat{\sigma}_{a \rightarrow X}$ where we factorize the complex non-perturbative QCD interactions into the Parton Distribution Functions (PDFs) $f_{a / A}$ and the theoretical partonic cross section $\widehat{\sigma}_{a \rightarrow X}$. Here, $\sigma_{A \rightarrow X}$ is the physically measurable cross section, and $A$ can represent either a proton or a nucleon.

As we push our calculations to higher precision and into extreme kinematic regions (such as low$Q^{2}$ or hi/low- $x$ ) we may encounter a variety of phenomena such as those illustrated schematically in Fig.1. While many of these phenomena can be neglected at lower orders and lower precision, if

${ }^{\star}$ Based on work in collaboration with D. B. Clark, E. Godat, T. Ježo, C. Keppel, K. Kovařík, J.G. Morfín, P. Nadolsky, J.F. Owens, \& J.Y. Yu. This work is supported in part by the U.S. Department of Energy under grant DE-FG02-04ER41299, and by Projet international de cooperation scientifique PICS05854 between France and the U.S.

a e-mail: kusina@lpsc.in2p3.fr

be-mail: flyonnet@smu.edu

ce-mail: olness@smu.edu

$\mathrm{d}^{\text {Presenter }}$

e-mail: schien@1psc.in2p3.fr

${ }^{1}$ In this short presentation we provide only an abbreviated set of references.

${ }^{2}$ What QCD Tells Us About Nature - and Why We Should Listen, by Frank Wilczek.[1] 
we are to properly analyze the new generation of high-precision high-statistics experiments, we must consider the potential impact of these manifestations of the QCD theory.

The recent performance of the LHC has exceeded expectations and produced an unprecedented number of events to be analyzed. However, to advance our understanding of these processes and the inherent aspects of the QCD theory requires both improved theoretical tools and complementary data from other experiments such as an Electron Ion Collider (EIC) and Large Hadron electron Collider (LHeC). In the same manner that the combination of the HERA $e p$ and Tevatron $p \bar{p}$ data provided maximum knowledge of the underlying physics, the combination of the LHC and future lepton-hadron data can further expand our search for new physics and our understanding of QCD.

To carry out this program requires both the above data, and also improved theoretical tools to perform the analysis. We briefly review some of the recent progress, and identify areas where additional effort is required.

\subsection{Nuclear PDFs}

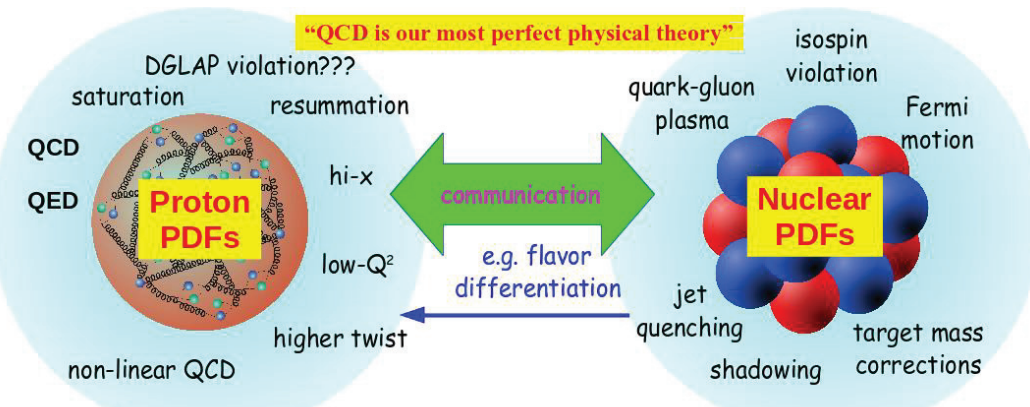

Figure 1. We schematically represent the variety of phenomena which may enter when considering proton and nuclear PDFs at high precision. We emphasize that the nuclear PDFs and corrections are important for flavor differentiation.

Historically, the nuclear PDFs (nPDFs) and the associated nuclear correction factors have played a key role in the flavor differentiation of the proton PDFs. The common processes used to extract the proton PDFs were Deeply Inelastic Scattering (DIS), Drell-Yan (DY) lepton-hadron scattering, and jet production. These processes involve different linear combinations of the partonic flavors, and the neutrino-nucleon DIS processes are especially valuable as we can extract the four quantities $\left\{F_{2}^{\nu N}\right.$, $\left.F_{2}^{\bar{\nu} N}, F_{3}^{\nu N}, F_{3}^{\bar{\nu} N}\right\}$. Due to the small neutrino cross section, these measurements are usually performed on heavy nuclear targets; hence, a detailed understanding of the nPDFs and their corrections are essential to constraining the flavor structure of both the nuclear and proton PDFs.

To comprehensively analyze both proton and nuclear data in a single unified framework, we have developed the nCTEQ project which can combine both data sets (including uncertainties) into the fit. The nCTEQ15 set of nPDFs[2] is illustrated in Fig.2, and this extends the CTEQ proton PDFs fits to include the nuclear dependence across the full $A$ range from proton up to ${ }^{208} \mathrm{~Pb}$.

\subsection{The Strange Quark}

To illustrate the challenges of fitting the nPDFs, in Fig.3 we display the effective nuclear correction factors $R$ on iron for neutral-current charged-lepton $\left(\ell^{ \pm}\right)$DIS and charged-current neutrino $(v, \bar{v})$ DIS. 

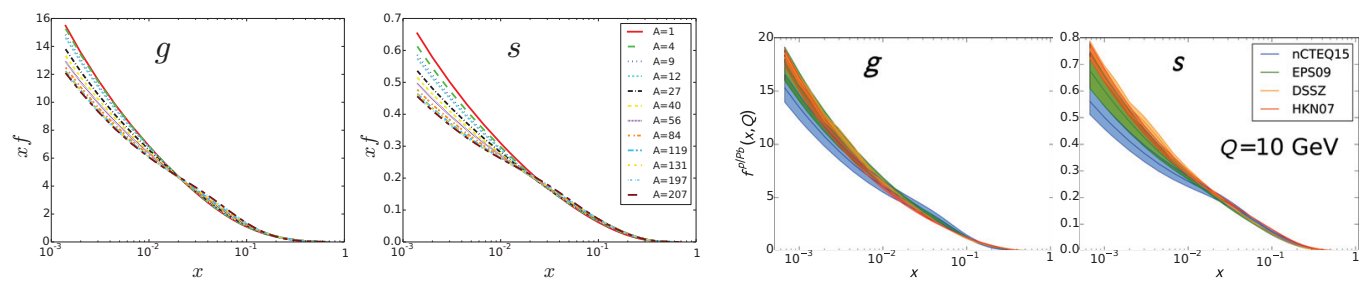

Figure 2. a) The nCTEQ15 PDFs for a range of nuclear $A$ values.[2] b) The nPDF uncertainty bands, together with HKN07,[3] EPS09,[4] and DSSZ.[5]
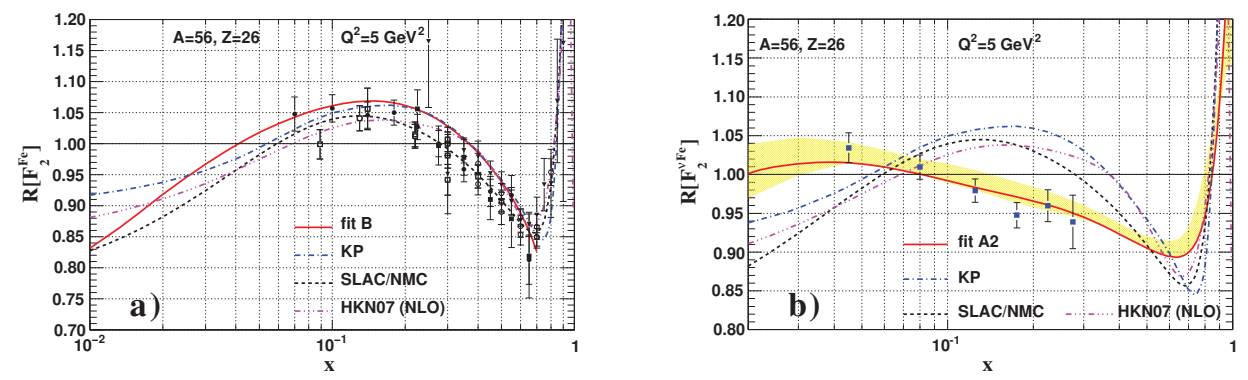

Figure 3. The computed nuclear correction ratio, $R=F_{2}^{F e} / F_{2}^{D}$ as a function of $x$. Figure-a) shows the fit using the neutral-current lepton $\left(\ell^{ \pm} N\right)$ DIS data (KP, SLAC/NMC, HKN07). Figure-b) shows the fit using the charged-current neutrino $(v N)$ DIS data; the data are from the NuTeV experiment. See Ref. [6]
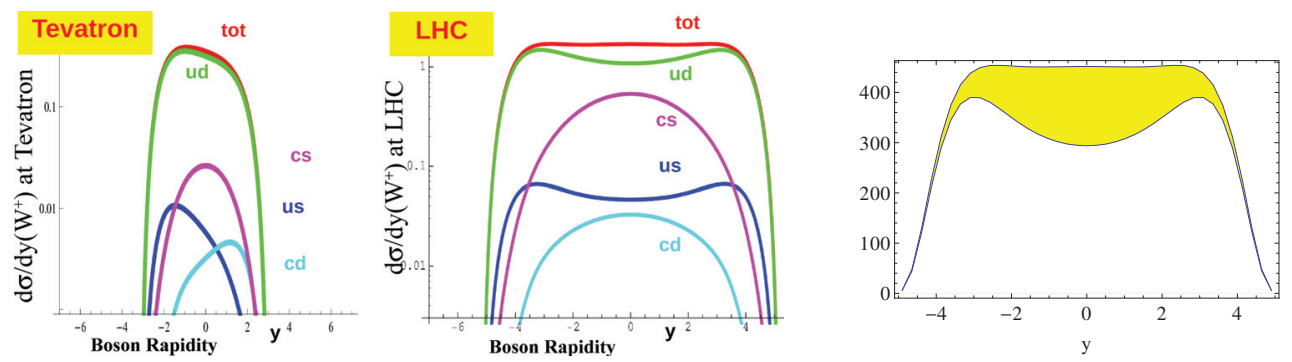

Figure 4. a) b) The leading-order (LO) differential cross section $(d \sigma / d y)$ for $W^{+}$production at the Tevatron $(2 \mathrm{TeV})$ and the LHC $(14 \mathrm{TeV})$ as a function of rapidity. The partonic contributions are also displayed for $\{u \bar{d}, c \bar{s}, u \bar{s}, c \bar{d}\}$. The vertical scales are logarithmic. c) The strange quark contribution (yellow) as a fraction of the total $d^{2} \sigma / d M / d y$ in $\mathrm{pb} / \mathrm{GeV}$ for $p p$ to $W^{+}$production at the LHC for $14 \mathrm{TeV}$ with CTEQ6.6 using the VRAP program at NNLO. C.f. Ref. [7] for details.

The $R$ values differ, and this uncertainty then feeds into the individual flavors such as the strange quark $s(x)$. Prior to the LHC data, our knowledge of $s(x)$ came primarily from $v \mathrm{Fe}$ DIS, so the uncertainty of the nuclear correction was an important consideration. Consequently, improvements in the nuclear correction factor $R$ will yield improved proton PDFs.

At the higher energy scales of the LHC, the (relatively) heavier parton flavors (such as the strange) play a more prominent role. This is illustrated in Fig.4 where we find the $c \bar{s} \rightarrow W^{+}$contribution is 
much larger than at the Tevatron (for example) where the $u \bar{d}$ process dominated. We can then turn this issue around and use the LHC data to impose constraints on the strange quark.[8]

In this instance, the combination of fixed-target $v N$ DIS data together with LHC $W / Z$ production data can improve constraints of both the strange PDF and the nuclear correction factors.

\subsection{Charm and Bottom Quark}
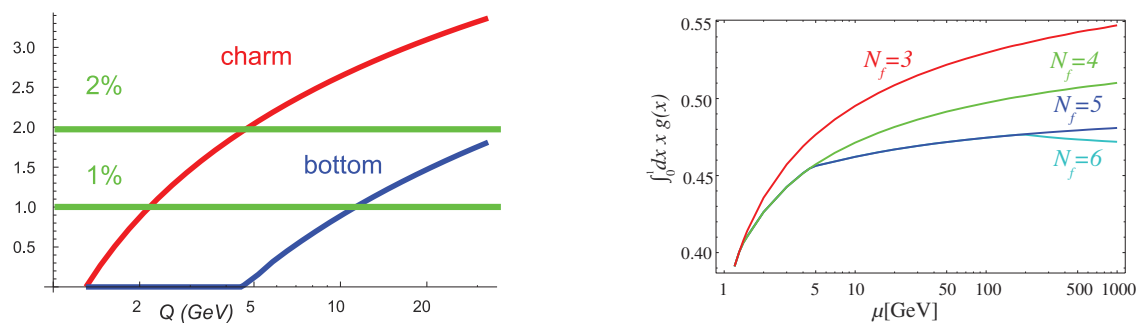

Figure 5. a) Momentum fraction of the charm and bottom PDFs as a function of the scale. b) Momentum fraction of the gluon for $N_{F}=\{3,4,5,6\}$ as a function of the scale.
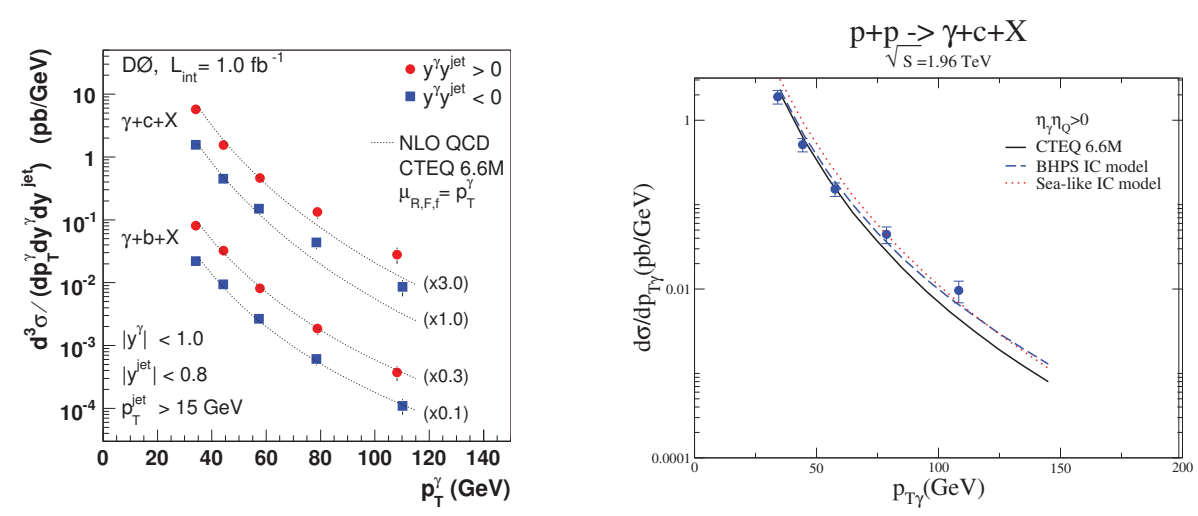

Figure 6. a) Cross section for associated production of $\gamma$ and $c / b$.[9] b) Same with an intrinsic charm component.[10]

Considering the heavier charm and bottom quarks, these enter the PDF for energy scales above the quark mass values; as the $c$ and $b$ PDFs are primarily produced by gluon splitting $(g \rightarrow c \bar{c}$ and $g \rightarrow b \bar{b}$ ), these affect the momentum fraction carried by the gluon (c.f., Fig.5).

While we generally assume that there is no primordial component of the $c$ and $b$ PDFs for energy scales below the quark mass, it has been postulated that there could be an additional "intrinsic" component which would be added to the usual "extrinsic" component (which comes from gluon splitting). There are a number of measurements where the "intrinsic" component could manifest itself; see Ref. [11] for a recent review.

Fig.6-a shows the data for $\gamma+c \rightarrow X$ is above the theory prediction at the largest $p_{T}^{\gamma}$ values; an additional "intrinsic" component (Fig.6-b) can shift the theory in the direction of the data at large $p_{T}^{\gamma}$. 
While this observation is intriguing, additional precision and analysis is required to definitively claim evidence of an "intrinsic" component.

On a related topic, recent theoretical work demonstrated that (to a good approximation) the evolution of the intrinsic heavy quark component is governed by non-singlet evolution equations which can be decoupled from the evolution of the remaining PDFs.[12] The observation allows an (approximate) intrinsic component to easily be included with any calculation without the need for a specially evolved PDF set; this greatly simplifies our ability to search for, and place constraints upon, intrinsic charm and bottom components of the nucleon.

\subsection{Higher Order Calculations}
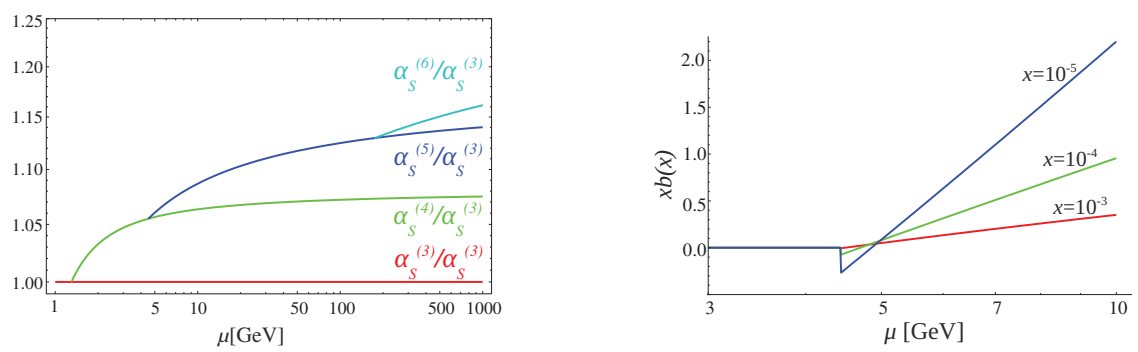

Figure 7. a) Ratios of $\alpha_{s}(\mu)$ for $N_{F}=\{3,4,5,6\}$. b) The discontinuity in the $b$-quark PDF $f_{b}(x, \mu)$ at NNLO $v s$. $\mu$ for $x=\left\{10^{-3}, 10^{-4}, 10^{-5}\right\}$.

Recent advances in the automation of higher order calculations have tremendously increased the breadth of processes which are available at NNLO and beyond. As we move to these higher orders, our PDFs and $\alpha_{s}(\mu)$ functions are discontinuous ${ }^{3}$ when we shift the number of flavors from $N_{F}$ to $N_{F}+1$; c.f. Fig.7. An elegant property of QCD is that despite the (fixed order) discontinuity of the PDFs and $\alpha_{s}$, the discontinuity of any physical quantity will systematically decrease order by order in perturbation theory; that is to say, at order $k$ we have the relation: $\sigma \simeq f_{N_{F}} \otimes \hat{\sigma}_{N_{F}}=f_{N_{F}+1} \otimes \hat{\sigma}_{N_{F}+1}+$ $O\left(\alpha_{s}^{k+1}\right)$. This is certainly a remarkable result.

\subsection{Incisive Analysis Tools}

Finally, as we advance our analysis methods it is important to develop new tools and metrics that can insightfully and effectively convey the essence of the physics. In Fig. 8 we display the correlation $\operatorname{cosine} \cos \phi$ vs. $x$ for a variety of experiments. The plot effectively conveys which experiments influence the PDFs in different $x$ regions, and how they are correlated. To emphasize this point we compare the $u$-valence (left) with the flipped $d$-valence figure (right); by flipping the figure we see that $u$-valence and $d$-valence are anti-correlated. Thus, as the fit works to find a minimum $\chi^{2}$ value, it has to balance these experimental data sets which are pulling the $u$-valence and $d$-valence in opposite directions.

\footnotetext{
${ }^{3} \alpha_{s}(\mu)$ has discontinuities at order $\alpha_{s}(\mu)^{3}$, but these are not apparent in Fig.7-a.
} 

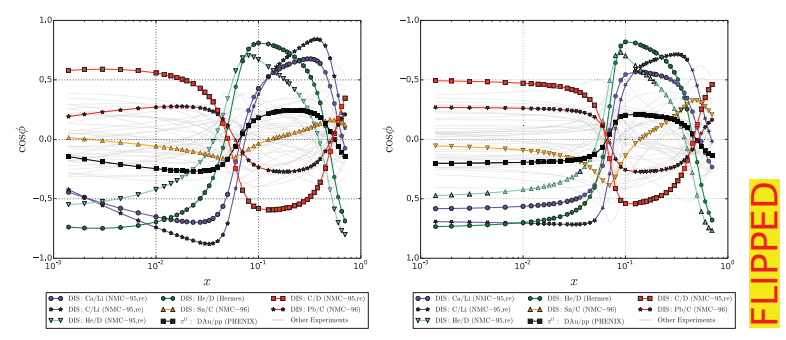

Figure 8. Correlation measures ( $\cos \phi$ ) for $u$-valence (left) and $d$-valence (right) for lead at $Q=10 \mathrm{GeV}$. Eight selected experiments are highlighted with symbols. To emphasize the anti-correlation between $u$-valence and $d$-valence we have flipped the $d$-valence plot vertically. See Ref.[2] for the original figures and additional details.

\subsection{Conclusions}

Recent theoretical developments provide improved precision for both proton and nuclear targets across a wide kinematic range. Further improvements will rely on a combination of additional theoretical advances, and a broad range of experimental measurements. The prospect of future data from EIC and $\mathrm{LHeC}$ facilities would be a valuable complement to the LHC and advance both our search for new physics and our understanding of QCD.

\section{References}

[1] F. Wilczek, Nucl. Phys. A 663, 3 (2000) [hep-ph/9907340].

[2] K. Kovarik et al., arXiv:1509.00792 [hep-ph].

[3] M. Hirai, S. Kumano and T.-H. Nagai, Phys. Rev. C 76, 065207 (2007) doi:10.1103/PhysRevC.76.065207 [arXiv:0709.3038 [hep-ph]].

[4] K. J. Eskola, H. Paukkunen and C. A. Salgado, JHEP 0904, 065 (2009) doi:10.1088/11266708/2009/04/065 [arXiv:0902.4154 [hep-ph]].

[5] D. de Florian, R. Sassot, P. Zurita and M. Stratmann, Phys. Rev. D 85, 074028 (2012) doi:10.1103/PhysRevD.85.074028 [arXiv:1112.6324 [hep-ph]].

[6] K. Kovarik, I. Schienbein, F. I. Olness, J. Y. Yu, C. Keppel, J. G. Morfin, J. F. Owens and T. Stavreva, Phys. Rev. Lett. 106, 122301 (2011) doi:10.1103/PhysRevLett.106.122301 [arXiv:1012.0286 [hep-ph]].

[7] A. Kusina et al., Phys. Rev. D 85, 094028 (2012) doi:10.1103/PhysRevD.85.094028 [arXiv:1203.1290 [hep-ph]].

[8] G. Aad et al. [ATLAS Collaboration], Phys. Rev. Lett. 109, 012001 (2012) doi:10.1103/PhysRevLett.109.012001 [arXiv:1203.4051 [hep-ex]].

[9] D. Duggan, arXiv:0906.0136 [hep-ex].

[10] T. P. Stavreva and J. F. Owens, Phys. Rev. D 79, 054017 (2009) doi:10.1103/PhysRevD.79.054017 [arXiv:0901.3791 [hep-ph]].

[11] S. J. Brodsky, A. Kusina, F. Lyonnet, I. Schienbein, H. Spiesberger and R. Vogt, Adv. High Energy Phys. 2015, 231547 (2015) doi:10.1155/2015/231547 [arXiv:1504.06287 [hep-ph]].

[12] F. Lyonnet, A. Kusina, T. Ježo, K. Kovarík, F. Olness, I. Schienbein and J. Y. Yu, JHEP 1507, 141 (2015) doi:10.1007/JHEP07(2015)141 [arXiv:1504.05156 [hep-ph]]. 